\title{
Stationary Bootstrapping for the Nonparametric AR-ARCH Model
}

\author{
Dong Wan Shin ${ }^{a}$, Eunju Hwang ${ }^{1, b}$ \\ ${ }^{a}$ Department of Statistics, Ewha Womans University, Korea; \\ ${ }^{b}$ Department of Applied Statistics, Gachon University, Korea
}

\begin{abstract}
We consider a nonparametric $\mathrm{AR}(1)$ model with nonparametric $\mathrm{ARCH}(1)$ errors. In order to estimate the unknown function of the ARCH part, we apply the stationary bootstrap procedure, which is characterized by geometrically distributed random length of bootstrap blocks and has the advantage of capturing the dependence structure of the original data. The proposed method is composed of four steps: the first step estimates the AR part by a typical kernel smoothing to calculate AR residuals, the second step estimates the ARCH part via the Nadaraya-Watson kernel from the AR residuals to compute ARCH residuals, the third step applies the stationary bootstrap procedure to the ARCH residuals, and the fourth step defines the stationary bootstrapped NadarayaWatson estimator for the ARCH function with the stationary bootstrapped residuals. We prove the asymptotic validity of the stationary bootstrap estimator for the unknown ARCH function by showing the same limiting distribution as the Nadaraya-Watson estimator in the second step.
\end{abstract}

Keywords: stationary bootstrap, $\mathrm{ARCH}$, nonparametric regression, consistency

\section{Introduction}

Diverse models have been considered for trends and volatilities of the time series to analyze financial or economic time series. A simple model is the autoregressive-generalized autoregressive conditional heteroscedastic (AR-GARCH) model. Among many variations of the AR-GARCH models, some people considered nonparametric trend and volatility models, i.e., nonparametric AR-ARCH models, for which estimations and large sample theories are available in Robinson (1983), Hardle and Tsybakov (1997), Bossaerts et al. (1996) and Hafner (1998).

Bootstrap methods are applied to improve accuracies of finite sample distributions of estimators or test statistics over those based on large sample theories. For trend-volatility models, Franke et al. $(2002,2004)$ considered the i.i.d. bootstrapping for kernel smoothing and volatility function in nonparametric first-order AR time series model with conditionally heteroscedastic errors. Goncalves and Kilian (2007) investigated wild bootstrap inference for $\operatorname{AR}(\infty)$ processes with conditional heteroskedasticity in error, and Kreiss et al. (2008) studied bootstrap tests for nonparametric regression models and applied the results to ARCH models. Shimizu (2013) applied the residual and wild bootstrap to the quasi-maximum likelihood estimators for parametric ARMA-GARCH model while Shimizu (2014) dealt with the residual and wild bootstrap for nonparametric AR-nonparametric

\footnotetext{
This research was supported by the Gachon University research fund of 2014 (GCU-2014-0128).

${ }^{1}$ Corresponding author: Department of Applied Statistics, Gachon University, Seongnam 13120, Korea.

E-mail: ehwang@gachon.ac.kr
}

Published 30 September 2015 / journal homepage: http://csam.or.kr

(c) 2015 The Korean Statistical Society, and Korean International Statistical Society. All rights reserved. 
ARCH model. Shimizu (2013) demonstrated the cases where bootstrap does not work for heteroscedastic time series models.

Diverse dependent structures are imposed on the observation by both trend and volatility for data from trend-volatility models. Therefore, block bootstrapping may be a better choice because the bootstrap sampling should capture the dependence structure of the original data in order for the bootstrap sample to have the same dependence structure as the observation, instead of i.i.d. bootstrapping and wild bootstrapping. For example, volatility clustering features prevalent in log-returns of financial assets such as stock prices and foreign exchange rates and in macroeconomic variables are reserved in block bootstrap samples but not in i.i.d bootstrap samples. Kreiss and Paparoditis (2011) and references provide a review of the bootstrap methods for dependent data.

We apply the stationary bootstrap method in this work as a bootstrap applicability for the ARCH model. The stationary bootstrap proposed by Politis and Romano (1994) is characterized by resampling blocks of geometrically distributed random length. As recent stationary bootstrap works, we mention Swensen (2003), Paparoditis and Politis (2005), Parker et al. (2006), and Shin and Hwang (2013) for inference in nonstationary time series such as unit root tests and cointegration analysis; and Hwang and Shin (2011) and Hwang and Shin (2013a, 2013b) for realized volatilities of log-returns of financial assets.

In this paper, the nonparametric ARCH regression model is considered and studied for asymptotic validity by means of the stationary bootstrap. We first estimate the AR part by a typical kernel smoothing and to calculate AR residuals in the nonparametric AR(1)-nonparametric ARCH(1) model, the second step estimates the ARCH part via the Nadaraya-Watson kernel from the AR residuals to compute ARCH residuals, the third step applies the stationary bootstrap procedure to the ARCH residuals and the stationary bootstrapped Nadaraya-Watson estimator in the final step is defined by stationary bootstrapped residuals. We prove asymptotic validity of the stationary bootstrap estimator for the unknown ARCH function by showing that it has the same limiting distribution as the Nadaraya-Watson estimator.

Our result is an extension of Shimizu (2014), who showed the weak consistency of the NadarayaWatson estimator for the ARCH part as well as proposed the residual and wild bootstrap estimators in the nonparametric AR-nonparametric ARCH model. However, Shimizu (2014)'s bootstrap methods are involved with i.i.d. sample in generating bootstrap processes, which may not preserve the dependence structure of the original data versus block bootstrap methods (such as stationary bootstrap) that capture the dependence structure.

This paper is organized as follows. In Section 2, the model, the assumptions and the existing theories are stated. In Section 3, the main result is presented along with description of the stationary bootstrap procedure. The conclusion is given in Section 4. Proof is reported in Appendix.

\section{Model, Assumptions and Existing Theories}

Let $\left\{X_{t}\right\}$ follow a nonparametric $\mathrm{AR}(1)$ model with nonparametric $\mathrm{ARCH}(1)$ errors:

$$
X_{t}=m\left(X_{t-1}\right)+\varepsilon_{t} \quad \text { and } \quad \varepsilon_{t}=\sigma\left(\varepsilon_{t-1}\right) \eta_{t},
$$

where $\left\{\eta_{t}\right\}$ is a sequence of i.i.d. random variables with mean 0 , variance 1 and $E\left[\eta_{t}^{4}\right]=: \kappa<\infty, m(\cdot)$ and $\sigma(\cdot)$ are unknown smooth functions. Our main interest is estimating $\sigma(\cdot)$. The $\mathrm{ARCH}(1)$ error in (2.1) can be expressed as

$$
\varepsilon_{t}^{2}=\sigma^{2}\left(\varepsilon_{t-1}\right)+\sqrt{\kappa-1} \sigma^{2}\left(\varepsilon_{t-1}\right) u_{t},
$$


where

$$
u_{t}=\frac{\eta_{t}^{2}-1}{\sqrt{\kappa-1}}
$$

with $E\left[u_{t}\right]=0, E\left[u_{t}^{2}\right]=1$. In the virtual case that $\left\{\varepsilon_{t}: t=0,1, \ldots, n\right\}$ are known, the NadarayaWatson (NW) estimator of $\sigma^{2}(\cdot)$ with bandwidth $h$ and kernel function $K_{h}(\cdot)$ is given by

$$
\hat{\sigma}_{h}^{2}(e)=\frac{\sum_{i=1}^{n} \varepsilon_{i}^{2} K_{h}\left(e-\varepsilon_{i-1}\right)}{\sum_{j=1}^{n} K_{h}\left(e-\varepsilon_{j-1}\right)} .
$$

Asymptotic normality of $\hat{\sigma}_{h}^{2}(e)$ was developed by Franke et al. (2002).

In case that $\left\{\varepsilon_{t}: t=0,1, \ldots, n\right\}$ are unknown, Shimizu (2014) considered a two-step estimation method. Assume a data set $\left\{X_{1}, X_{2}, \ldots, X_{n}\right\}$ is available. First, $m(\cdot)$ in the AR part of the model (2.1) is estimated by the typical kernel smoothing with bandwidth $k$. The kernel smoothing estimator is denoted by $\hat{m}_{k}(\cdot)$ :

$$
\hat{m}_{k}(x)=\frac{\sum_{i=1}^{n} X_{i} K_{k}\left(x-X_{i-1}\right)}{\sum_{j=1}^{n} K_{k}\left(x-X_{j-1}\right)} .
$$

Second, the residuals $\hat{\varepsilon}_{t}:=X_{t}-\hat{m}_{k}\left(X_{t-1}\right)$ are used to estimate the ARCH part and provide the NW estimator of $\sigma^{2}(\cdot)$ :

$$
\tilde{\sigma}_{h}^{2}(e)=\frac{\sum_{i=1}^{n} \hat{\varepsilon}_{i}^{2} K_{h}\left(e-\hat{\varepsilon}_{i-1}\right)}{\sum_{j=1}^{n} K_{h}\left(e-\hat{\varepsilon}_{j-1}\right)} .
$$

Asymptotic normality of $\tilde{\sigma}_{h}^{2}(e)$ was given by Shimizu (2014), under the following conditions (A1)-(A8) below.

(A1): The distribution of the i.i.d. innovations $\eta_{t}$ possesses a Lebesgue density $p_{\eta}$, which satisfies $\inf _{e \in C} p_{\eta}(e)>0$ for all compact sets $C$.

(A2): $\sigma$ and $\sigma^{-1}$ are bounded on compact sets and $\lim \sup _{|e| \rightarrow \infty} E\left|\sigma(e) \eta_{1}\right| /|e|<1$.

Conditions in (A1) and (A2) ensure that the process $\left\{\varepsilon_{t}\right\}$ is stationary and geometrically ergodic and its stationary distribution $\pi$ possesses a strictly positive Lebesgue density denoted by $p$. From (2.1) we obtain

$$
p(e)=\int_{\mathbb{R}} \frac{1}{\sigma(u)} p_{\eta}\left(\frac{e}{\sigma(u)}\right) p(u) d u .
$$

(A3): $\sigma$ is twice continuously differentiable and $\sigma^{\prime}, \sigma^{\prime \prime}$ are bounded. There exists $\sigma_{0}>0$ such that $\sigma(e) \geq \sigma_{0}$ for all $e \in \mathbb{R}$.

(A4): $p_{\eta}$ is twice continuously differentiable. $p_{\eta}, p_{\eta}^{\prime}$ and $p_{\eta}^{\prime \prime}$ are bounded and $\sup _{e \in \mathbb{R}}\left|e p_{\eta}^{\prime}(e)\right|<\infty$.

(A5): The bandwidth $h$ of kernel estimation for the ARCH part satisfies $h \rightarrow 0, n h^{4} \rightarrow \infty, n h^{5} \rightarrow B^{2}$ for some $B$ as $n \rightarrow \infty$. 
(A6): The kernel function $K$ for the ARCH part has compact support $[-1,1] . K$ is symmetric, nonnegative and three times continuously differentiable with $K(1)=K^{\prime}(1)=0$ and $\int K(u) d u=1$.

For the AR part we need the following assumptions on the kernel smoothing estimator $\hat{m}_{k}(\cdot)$ with the bandwidth $k$.

(A7): $\sup _{e \in D}\left|\hat{m}_{k}(e)-m(e)\right|=O_{p}(\sqrt{\log n /(n k)})$ where $D$ denotes any compact subset of $\mathbb{R}$.

(A8): $k \rightarrow 0$ and $k \sim n^{-\gamma}$ for some $0<\gamma<\min \{1 / 5,1-8 \alpha\}$ with some $0<\alpha \leq 1 / 9$ as $n \rightarrow \infty$.

The following results are asymptotic normalities of $\hat{\sigma}_{h}^{2}(e)$ and $\tilde{\sigma}_{h}^{2}(e)$ by Franke et al. (2002) and by Shimizu (2014), respectively.

Theorem 1. (Franke et al., 2002) Assume (A1)-(A8) above. For all $e \in \mathbb{R}$, we have

$$
\sqrt{n h}\left(\hat{\sigma}_{h}^{2}(e)-\sigma^{2}(e)\right) \stackrel{\mathrm{d}}{\longrightarrow} N\left(\frac{b(e)}{p(e)}, \frac{v^{2}(e)}{p^{2}(e)}\right) \quad \text { as } n \rightarrow \infty,
$$

where

$$
\begin{aligned}
b(e) & =B\left(p^{\prime}(e)\left(\sigma^{2}\right)^{\prime}(e)+\frac{1}{2} p(e)\left(\sigma^{2}\right)^{\prime \prime}(e)\right) \int u^{2} K(u) d u, \\
v^{2}(e) & =(\kappa-1) p(e) \sigma^{4}(e) \int K^{2}(u) d u .
\end{aligned}
$$

Theorem 2. (Shimizu, 2014) Assume (A1)-(A8) above. For all e $\in \mathbb{R}$, we have

$$
\sqrt{n h}\left(\tilde{\sigma}_{h}^{2}(e)-\sigma^{2}(e)\right) \stackrel{\mathrm{d}}{\longrightarrow} N\left(\frac{b(e)}{p(e)}, \frac{v^{2}(e)}{p^{2}(e)}\right) \quad \text { as } n \rightarrow \infty .
$$

\section{Stationary Bootstrap}

In this section, we apply the stationary bootstrap method to define a bootstrap estimator of $\sigma^{2}(\cdot)$ and present the asymptotic validity of the stationary bootstrap.

\subsection{Stationary bootstrap procedure}

The stationary bootstrapping will be applied to a residual set $\left\{\tilde{u}_{1}, \ldots, \tilde{u}_{n}\right\}$, defined in the next subsection. For notational simplicity, we use $u_{t}$ for $\tilde{u}_{t}$ in this subsection. We describe how to construct stationary bootstrap sample $u_{1}^{*}, \ldots, u_{n}^{*}$ from $u_{1}, \ldots, u_{n}$. First we define a new time series $\left\{u_{n i}: i \geq 1\right\}$ by a periodic extension of the observed data set as follows. For each $i \geq 1$, define $u_{n i}:=u_{j}$ where $j$ is such that $i=q n+j$ for some $q$. The sequence $\left\{u_{n i}: i \geq 1\right\}$ is obtained by wrapping the data $u_{1}, \ldots, u_{n}$ around a circle, and re-labelling them as $u_{n 1}, u_{n 2}, \ldots$ Next, for a positive integer $\ell$, define the blocks $B(i, \ell), i \geq 1$ as $B(i, \ell)=\left\{u_{n i}, \ldots, u_{n(i+\ell-1)}\right\}$ consisting of $\ell$ observations starting from $u_{n i}$. Bootstrap observations under the stationary bootstrap method are obtained by selecting a random number of blocks from collection $\{B(i, \ell): i \geq 1, \ell \geq 1\}$. To do this, we generate random variables $I_{1}, \ldots, I_{n}$ and $L_{1}, \ldots, L_{n}$ as follows: (i) $I_{1}, \ldots, I_{n}$ are i.i.d. discrete uniform on $\{1, \ldots, n\}: P\left(I_{1}=i\right)=1 / n$, for $i=1, \ldots, n$, (ii) $L_{1}, \ldots, L_{n}$ are i.i.d. random variables having the geometric distribution with a parameter $\rho \in(0,1): P\left(L_{1}=\ell\right)=\rho(1-\rho)^{\ell-1}$ for $\ell=1,2, \ldots$, where $\rho=\rho(n)$ depends on the sample size $n$, and (iii) the collections $\left\{I_{1}, \ldots, I_{n}\right\}$ and $\left\{L_{1}, \ldots, L_{n}\right\}$ are independent. Now, a pseudo-time series 
$u_{1}^{*}, \ldots, u_{n}^{*}$ is generated in the following way. Let $\tau=\inf \left\{k \geq 1: L_{1}+\cdots+L_{k} \geq n\right\}$. Then select $\tau$ blocks $B\left(I_{1}, L_{1}\right), \ldots, B\left(I_{\tau}, L_{\tau}\right)$. Note that there are $L_{1}+\cdots+L_{\tau}$ elements in the resampled blocks $B\left(I_{1}, L_{1}\right), \ldots, B\left(I_{\tau}, L_{\tau}\right)$. Arranging these elements in a series and deleting the last $L_{1}+\cdots+L_{\tau}-n$ elements, we obtain the bootstrap observations $u_{1}^{*}, \ldots, u_{n}^{*}$.

\subsection{Stationary bootstrap NW estimator}

The stationary bootstrap procedure is applied to estimate $\sigma^{2}(\cdot)$. In order to define the stationary bootstrap estimator of $\sigma^{2}(\cdot)$, we follow four steps:

Step 1: Calculate residuals $\hat{\varepsilon}_{t}$ with the kernel smoothing estimator $\hat{m}_{k}(x)$;

$$
\hat{\varepsilon}_{t}=X_{t}-\hat{m}_{k}\left(X_{t-1}\right), \quad t=1,2, \ldots, n,
$$

and compute the NW estimator $\tilde{\sigma}_{g}^{2}(\cdot)$ with a bandwidth $g$ :

$$
\tilde{\sigma}_{g}^{2}(e)=\frac{\sum_{i=1}^{n} \hat{\varepsilon}_{i}^{2} K_{g}\left(e-\hat{\varepsilon}_{i-1}\right)}{\sum_{j=1}^{n} K_{g}\left(e-\hat{\varepsilon}_{j-1}\right)} .
$$

Step 2: Compute

$$
\hat{\eta}_{t}=\frac{\hat{\varepsilon}_{t}}{\tilde{\sigma}_{g}\left(\hat{\varepsilon}_{t-1}\right)}, \quad \hat{\kappa}=\frac{1}{n} \sum_{t=1}^{n} \hat{\eta}_{t}^{4}
$$

and

$$
\hat{u}_{t}=\frac{\hat{\eta}_{t}^{2}-1}{\sqrt{\hat{\kappa}-1}}
$$

and standardize $\hat{u}_{t}$ to get

$$
\tilde{u}_{t}=\frac{\hat{u}_{t}-\hat{\mu}}{\hat{\sigma}} \quad \text { with } \hat{\mu}=\frac{1}{n} \sum_{t=1}^{n} \hat{u}_{t}, \quad \hat{\sigma}^{2}=\frac{1}{n} \sum_{t=1}^{n}\left(\hat{u}_{t}-\hat{\mu}\right)^{2} .
$$

Step 3: Apply the stationary bootstrap procedure to $\left\{\tilde{u}_{t}: t=1,2, \ldots, n\right\}$ to generate the stationary bootstrap sample $\left\{\tilde{u}_{t}^{*}: t=1,2, \ldots, n\right\}$. Note that

$$
\tilde{u}_{t}^{*}=\frac{1}{\hat{\sigma}}\left[\frac{\hat{\eta}_{t}^{* 2}-1}{\sqrt{\hat{\kappa}-1}}-\hat{\mu}\right],
$$

where $\left\{\hat{\eta}_{t}^{* 2}\right\}$ is the stationary bootstrap sample generated from $\left\{\hat{\eta}_{t}^{2}\right\}$.

Step 4: Following (2.2), compute

$$
\hat{\varepsilon}_{t}^{* 2}=\tilde{\sigma}_{g}^{2}\left(\hat{\varepsilon}_{t-1}\right)+\sqrt{\hat{\kappa}-1} \tilde{\sigma}_{g}^{2}\left(\hat{\varepsilon}_{t-1}\right) \tilde{u}_{t}^{*}, \quad t=1,2, \ldots, n,
$$

and the NW estimator of the stationary bootstrap version

$$
\tilde{\sigma}_{h}^{* 2}(e)=\frac{\sum_{i=1}^{n} \hat{\varepsilon}_{i}^{* 2} K_{h}\left(e-\hat{\varepsilon}_{i-1}\right)}{\sum_{j=1}^{n} K_{h}\left(e-\hat{\varepsilon}_{j-1}\right)} .
$$




\subsection{Asymptotic validity}

Now we present the asymptotic validity of the stationary bootstrap NW estimator $\tilde{\sigma}_{h}^{* 2}(\cdot)$. In addition to (A1)-(A8), two additional conditions are needed for the consistency of the stationary bootstrap.

(A9): The bandwidth $g$ of kernel estimator $\tilde{\sigma}_{g}^{2}(\cdot)$ in Step 1 satisfies $g \sim n^{-\alpha}$ for some $0<\alpha \leq 1 / 9$ as $n \rightarrow \infty$.

(A10): The parameter $\rho$ of the geometrically distributed length of the stationary bootstrap block satisfies $n \rho h^{1+2 / \delta} \rightarrow \infty$ for some $\delta>0$.

Theorem 3. Assume (Al)-(A10) above. For all e $\in \mathbb{R}$, we have, as $n \rightarrow \infty$

$$
\sup _{x \in \mathbb{R}}\left|P^{*}\left(\sqrt{n h}\left[\tilde{\sigma}_{h}^{* 2}(e)-\tilde{\sigma}_{g}^{2}(e)\right] \leq x\right)-P\left(\sqrt{n h}\left[\tilde{\sigma}_{h}^{2}(e)-\sigma^{2}(e)\right] \leq x\right)\right| \stackrel{\mathrm{p}}{\longrightarrow} 0 .
$$

Remark 1. An alternative way to use the stationary bootstrap procedure is that we apply the procedure directly to the residuals $\left\{\hat{\varepsilon}_{t}: t=1, \ldots, n\right\}$ in Step 1 to get the bootstrap sample $\left\{\hat{\varepsilon}_{t}^{*}: t=1, \ldots, n\right\}$. The stationary bootstrap estimator of $\sigma^{2}(\cdot)$ can be defined as

$$
\hat{\sigma}_{h}^{* 2}(e)=\frac{\sum_{i=1}^{n} \hat{\varepsilon}_{i}^{* 2} K_{h}\left(e-\hat{\varepsilon}_{i-1}^{*}\right)}{\sum_{j=1}^{n} K_{h}\left(e-\hat{\varepsilon}_{j-1}^{*}\right)}
$$

just as in the stationary bootstrap kernel estimator of nonlinear autoregressive model in Equation (4) of Hwang and Shin (2011). The consistency result of $\hat{\sigma}_{h}^{* 2}(e)$ can be shown by the similar arguments to those in the proof of Hwang and Shin (2011).

\section{Conclusion}

We developed a stationary bootstrapping procedure for a nonparametric NW estimator in AR-ARCH model. Large sample validity of the proposed bootstrap procedure is proved in a rigorous manner. The asymptotic validity will enable us to construct statistical inference methods such as confidence intervals and hypothesis testing. It would be worth to compare the bootstrap methods with other non-bootstrap methods in a Monte-Carlo experiment. This issue may be a topic for future studies.

\section{Appendix:}

Proof of Theorem 3: According to Theorem 2, it suffices to show that $\sqrt{n h}\left[\tilde{\sigma}_{h}^{* 2}(e)-\tilde{\sigma}_{g}^{2}(e)\right]$ has the same asymptotic normal distribution as that in Theorem 2.

Letting $K_{h, i}=K_{h}\left(e-\hat{\varepsilon}_{i-1}\right)$ for just notational simplicity, we write

$$
\sqrt{n h}\left[\tilde{\sigma}_{h}^{* 2}(e)-\tilde{\sigma}_{g}^{2}(e)\right]=\frac{\sqrt{\frac{h}{n}} \sum_{i=1}^{n} K_{h, i} \sqrt{\hat{\kappa}-1} \tilde{\sigma}_{g}^{2}\left(\hat{\varepsilon}_{i-1}\right) \tilde{u}_{i}^{*}}{\frac{1}{n} \sum_{j=1}^{n} K_{h, j}}+\frac{\sqrt{\frac{h}{n}} \sum_{i=1}^{n} K_{h, i}\left(\tilde{\sigma}_{g}^{2}\left(\hat{\varepsilon}_{i-1}\right)-\tilde{\sigma}_{g}^{2}(e)\right)}{\frac{1}{n} \sum_{j=1}^{n} K_{h, j}} .
$$

The following two convergence results in (A.2) were given by Shimizu (2014); (see respectively (ii) in the proof of Theorem 2.2 of Shimizu $(2014$, p.65) and (ii) in the proof of Theorem 3.1 of Shimizu (2014, p.67)):

$$
\frac{1}{n} \sum_{j=1}^{n} K_{h, j} \stackrel{\mathrm{p}}{\longrightarrow} p(e) \quad \text { and } \quad \sqrt{\frac{h}{n}} \sum_{i=1}^{n} K_{h, i}\left(\tilde{\sigma}_{g}^{2}\left(\hat{\varepsilon}_{i-1}\right)-\tilde{\sigma}_{g}^{2}(e)\right) \stackrel{\mathrm{p}}{\longrightarrow} b(e) .
$$


Thus we may show that the numerator of the first term in (A.1), which is the stationary bootstrap part, converges in distribution conditionally given $\left\{X_{t}\right\}$ to $N\left(0, v^{2}(e)\right)$. We observe the numerator of the first term in (A.1).

Let

$$
\phi_{i}^{*}(e)=\sqrt{\frac{h}{n}} K_{h, i} \sqrt{\hat{\kappa}-1} \tilde{\sigma}_{g}^{2}\left(\hat{\varepsilon}_{i-1}\right) \tilde{u}_{i}^{*}, \quad i=1,2, \ldots, n .
$$

Since

$$
\begin{aligned}
\hat{\mu} & =\frac{1}{n} \sum_{i=1}^{n}\left[\frac{1}{\sqrt{\hat{\kappa}-1}}\left(\hat{\eta}_{i}^{2}-1\right)\right], \\
\phi_{i}^{*}(e) & =\frac{1}{\hat{\sigma}} \sqrt{\frac{h}{n}} K_{h, i} \tilde{\sigma}_{g}^{2}\left(\hat{\varepsilon}_{i-1}\right)\left[\hat{\eta}_{i}^{2 *}-1-\hat{\mu} \sqrt{\hat{\kappa}-1}\right]=\frac{1}{\hat{\sigma}} \sqrt{\frac{h}{n}} K_{h, i} \tilde{\sigma}_{g}^{2}\left(\hat{\varepsilon}_{i-1}\right)\left[\hat{\eta}_{i}^{2 *}-\overline{\eta^{2}}\right],
\end{aligned}
$$

where $\overline{\eta^{2}}=(1 / n) \sum_{i=1}^{n} \hat{\eta}_{i}^{2}$. Note that $E^{*}\left[\phi_{i}^{*}(e)\right]=0$.

It will be shown that $\sum_{i=1}^{n} \phi_{i}^{*}(e) \stackrel{\mathrm{d}^{*}}{\longrightarrow} N\left(0, v^{2}(e)\right)$. We have

$$
\operatorname{Var}^{*}\left(\sum_{i=1}^{n} \phi_{i}^{*}(e)\right)=n\left[R^{*}(0)+2 \sum_{i=1}^{n-1}\left(1-\frac{i}{n}\right) R^{*}(i)\right],
$$

where $R^{*}(i)=\operatorname{Cov}^{*}\left(\phi_{1}^{*}(e), \phi_{1+i}^{*}(e)\right)$, which is equal to

$$
\frac{1}{\hat{\sigma}^{2}} \frac{h}{n} K_{h, 1} K_{h, 1+i} \tilde{\sigma}_{g}^{2}\left(\hat{\varepsilon}_{0}\right) \tilde{\sigma}_{g}^{2}\left(\hat{\varepsilon}_{i}\right) E^{*}\left[\left(\hat{\eta}_{1}^{2 *}-\bar{\eta}^{2}\right)\left(\hat{\eta}_{1+i}^{2 *}-\overline{\eta^{2}}\right)\right] .
$$

Observe

$$
\begin{aligned}
& E^{*}\left[\left(\hat{\eta}_{1}^{2 *}-\bar{\eta}^{2}\right)\left(\hat{\eta}_{1+i}^{2 *}-\bar{\eta}^{2}\right)\right] \\
& =E^{*}\left[\left(\hat{\eta}_{1}^{2 *}-\bar{\eta}^{2}\right)\left(\hat{\eta}_{1+i}^{2 *}-\bar{\eta}^{2}\right) \mid L_{1}>i\right] P\left(L_{1}>i\right)+E^{*}\left[\left(\hat{\eta}_{1}^{2 *}-\bar{\eta}^{2}\right)\left(\hat{\eta}_{1+i}^{2 *}-\bar{\eta}^{2}\right) \mid L_{1} \leq i\right] P\left(L_{1} \leq i\right) \\
& =(1-\rho)^{i}\left[\hat{d}_{n}(i)+\hat{d}_{n}(n-i)\right],
\end{aligned}
$$

where $\hat{d}_{n}(i)=(1 / n) \sum_{j=1}^{n-i}\left(\hat{\eta}_{j}^{2}-\bar{\eta}^{2}\right)\left(\hat{\eta}_{j+i}^{2}-\bar{\eta}^{2}\right)$.

Then, letting $\hat{K}(i)=K_{h, 1} K_{h, 1+i} \tilde{\sigma}_{g}^{2}\left(\hat{\varepsilon}_{0}\right) \tilde{\sigma}_{g}^{2}\left(\hat{\varepsilon}_{i}\right)$,

$$
R^{*}(i)=\frac{1}{\hat{\sigma}^{2}} \frac{h}{n} \hat{K}(i)(1-\rho)^{i}\left[\hat{d}_{n}(i)+\hat{d}_{n}(n-i)\right]
$$

and thus

$$
\sum_{i=1}^{n-1}\left(1-\frac{i}{n}\right) R^{*}(i)=\frac{1}{\hat{\sigma}^{2}} \frac{h}{n} \sum_{i=1}^{n-1} \hat{K}(i) b_{n}(i) \hat{d}_{n}(i),
$$

where $b_{n}(i)=\{1-i /(n-1)\}(1-\rho)^{i}+\{i /(n-1)\}(1-\rho)^{n-1-i}$.

Let

$$
\phi_{i}(e)=\frac{1}{\hat{\sigma}} \sqrt{\frac{h}{n}} K_{h, i} \tilde{\sigma}_{g}^{2}\left(\hat{\varepsilon}_{i-1}\right)\left[\hat{\eta}_{i}^{2}-\bar{\eta}^{2}\right] .
$$


Therefore $\operatorname{Var}\left(\sum_{i=1}^{n} \phi_{i}(e)\right)=\operatorname{Var}\left(\sum_{i=1}^{n} 1 / \hat{\sigma} \sqrt{h / n} \sum_{i=1}^{n} K_{h, i} \hat{\varepsilon}_{i}^{2}\right) \rightarrow v^{2}(e)$ by Theorem 2 and its proof in Shimizu (2014). We can write $\lim _{n \rightarrow \infty} \operatorname{Var}\left(\sum_{i=1}^{n} \phi_{i}(e)\right)=R(0)+2 \sum_{i=1}^{\infty} R(i)$ with

$$
R(i)=\operatorname{Cov}\left(\phi_{1}(e), \phi_{1+i}(e)\right)=\frac{1}{\hat{\sigma}^{2}} \frac{h}{n} \operatorname{Cov}\left(K_{h, 1} \tilde{\sigma}_{g}^{2}\left(\hat{\varepsilon}_{0}\right)\left[\hat{\eta}_{1}^{2}-\bar{\eta}^{2}\right], K_{h, 1+i} \tilde{\sigma}_{g}^{2}\left(\hat{\varepsilon}_{i}\right)\left[\hat{\eta}_{1+i}^{2}-\bar{\eta}^{2}\right]\right) .
$$

Using the same arguments as that in Politis and Romano (1994) or Hwang and Shin (2012), the following two convergences in probability can be shown:

$$
\begin{aligned}
n\left|R^{*}(0)-R(0)\right|= & \frac{h}{\hat{\sigma}^{2}}\left|\frac{1}{n} \sum_{j=1}^{n}\left(K_{h, 1} \tilde{\sigma}_{g}^{2}\left(\hat{\varepsilon}_{0}\right)\left[\hat{\eta}_{j}^{2}-\bar{\eta}^{2}\right]\right)^{2}-\operatorname{Var}\left(K_{h, 1} \tilde{\sigma}_{g}^{2}\left(\hat{\varepsilon}_{0}\right)\left[\hat{\eta}_{1}^{2}-\bar{\eta}^{2}\right]\right)\right| \\
\leq & \frac{h}{\hat{\sigma}^{2}}\left|\frac{1}{n} \sum_{j=1}^{n}\left(K_{h, 1} \tilde{\sigma}_{g}^{2}\left(\hat{\varepsilon}_{0}\right)\left[\hat{\eta}_{j}^{2}-\bar{\eta}^{2}\right]\right)^{2}-\frac{1}{n} \sum_{j=1}^{n}\left(K_{h, j} \tilde{\sigma}_{g}^{2}\left(\hat{\varepsilon}_{j-1}\right)\left[\hat{\eta}_{j}^{2}-\bar{\eta}^{2}\right]\right)^{2}\right| \\
& +\frac{h}{\hat{\sigma}^{2}}\left|\frac{1}{n} \sum_{j=1}^{n}\left(K_{h, j} \tilde{\sigma}_{g}^{2}\left(\hat{\varepsilon}_{j-1}\right)\left[\hat{\eta}_{j}^{2}-\overline{\eta^{2}}\right]\right)^{2}-\operatorname{Var}\left(K_{h, 1} \tilde{\sigma}_{g}^{2}\left(\hat{\varepsilon}_{0}\right)\left[\hat{\eta}_{1}^{2}-\overline{\eta^{2}}\right]\right)\right| \stackrel{\mathrm{p}}{\longrightarrow} 0,
\end{aligned}
$$

and

$$
\begin{aligned}
& n\left|\sum_{i=1}^{n-1}\left(1-\frac{i}{n}\right) R^{*}(i)-\sum_{i=1}^{\infty} R(i)\right| \\
& \leq \frac{h}{\hat{\sigma}^{2}} \mid \frac{1}{n} \sum_{i=1}^{n-1} b_{n}(i)\left\{\sum_{j=1}^{n-i} K_{h, 1} \tilde{\sigma}_{g}^{2}\left(\hat{\varepsilon}_{0}\right)\left[\hat{\eta}_{j}^{2}-\bar{\eta}^{2}\right] K_{h, 1+i} \tilde{\sigma}_{g}^{2}\left(\hat{\varepsilon}_{i}\right)\left[\hat{\eta}_{j+i}^{2}-\bar{\eta}^{2}\right]\right. \\
& \left.\quad-\sum_{j=1}^{n-i} K_{h, j} \tilde{\sigma}_{g}^{2}\left(\hat{\varepsilon}_{j-1}\right)\left[\hat{\eta}_{j}^{2}-\bar{\eta}^{2}\right] K_{h, j+i} \tilde{\sigma}_{g}^{2}\left(\hat{\varepsilon}_{j+i-1}\right)\left[\hat{\eta}_{j+i}^{2}-\bar{\eta}^{2}\right]\right\} \mid \\
& +\frac{h}{\hat{\sigma}^{2}}\left|\frac{1}{n} \sum_{i=1}^{n-1} b_{n}(i)\left\{\sum_{j=1}^{n-i} K_{h, j} \tilde{\sigma}_{g}^{2}\left(\hat{\varepsilon}_{j-1}\right)\left[\hat{\eta}_{j}^{2}-\bar{\eta}^{2}\right] K_{h, j+i} \tilde{\sigma}_{g}^{2}\left(\hat{\varepsilon}_{j+i-1}\right)\left[\hat{\eta}_{j+i}^{2}-\bar{\eta}^{2}\right]-C_{K}(i)\right\}\right| \\
& +\frac{h}{\hat{\sigma}^{2}}\left|\frac{1}{n} \sum_{i=1}^{n-1} b_{n}(i) C_{K}(i)-\sum_{i=1}^{n} C_{K}(i)\right| \stackrel{\mathrm{p}}{\longrightarrow} 0
\end{aligned}
$$

where $C_{K}(i)=\operatorname{Cov}\left(K_{h, 1} \tilde{\sigma}_{g}^{2}\left(\hat{\varepsilon}_{0}\right)\left[\hat{\eta}_{1}^{2}-\bar{\eta}^{2}\right], K_{h, 1+i} \tilde{\sigma}_{g}^{2}\left(\hat{\varepsilon}_{i}\right)\left[\hat{\eta}_{1+i}^{2}-\overline{\eta^{2}}\right]\right)$. Therefore we conclude that Var* $\left(\sum_{i=1}^{n} \phi_{i}^{*}(e)\right) \stackrel{\mathrm{p}}{\longrightarrow} v^{2}(e)$.

Now we establish the asymptotic normality of $\sum_{i=1}^{n} \phi_{i}^{*}(e)$. Let $M_{k}=L_{1}+\cdots+L_{k}$ for $k=$ $1, \ldots, \tau$. Note that $M_{\tau-1}<n \leq M_{\tau}$, and $\sum_{i=1}^{n} \phi_{i}^{*}(e)=\sum_{i=1}^{M_{\tau-1}} \phi_{i}^{*}(e)+\sum_{i=M_{\tau-1}+1}^{n} \phi_{i}^{*}(e)$. It is clear that $\sum_{i=M_{\tau-1}+1}^{n} \phi_{i}^{*}(e) \stackrel{\mathrm{p}^{*}}{\longrightarrow} 0$ as $n \rightarrow \infty$ because both $\sum_{i=1}^{M_{\tau-1}} \phi_{i}^{*}(e)$ and $\sum_{i=1}^{M_{\tau}} \phi_{i}^{*}(e)$ have the same limit.

We consider $\sum_{i=1}^{M_{\tau-1}} \phi_{i}^{*}(e)$. Let $\xi_{n, 1}^{*}=\sum_{i=1}^{M_{1}} \phi_{i}^{*}(e)$ and for $k=2, \ldots, \tau-1, \xi_{n, k}^{*}=\sum_{i=M_{k-1}+1}^{M_{k}} \phi_{i}^{*}(e)$. Then $\sum_{i=1}^{M_{\tau-1}} \phi_{i}^{*}(e)=\sum_{k=1}^{\tau-1} \xi_{n, k}^{*}$, and $\left\{\xi_{n, 1}^{*}, \ldots, \xi_{n, \tau-1}^{*}\right\}$ is a triangular array of independent random variables because of independence of $L_{1}, L_{2}, \ldots, L_{\tau-1}$. Lyapunov's central limit theorem is adiquate to show 
that, for some $\delta>0$

$$
\frac{1}{v_{\xi, \tau}^{2+\delta}} \sum_{k=1}^{\tau-1} E^{*}\left[\left|\xi_{n, k}^{*}\right|^{2+\delta}\right] \stackrel{\mathrm{p}}{\longrightarrow} 0 \quad \text { as } n \rightarrow \infty(\tau \rightarrow \infty),
$$

where $v_{\xi, \tau}=\left(\operatorname{Var}^{*}\left[\sum_{k=1}^{\tau-1} \xi_{n, k}^{*}\right]\right)^{1 / 2}$. It is obvious that $v_{\xi, \tau}^{2} \stackrel{\mathrm{p}}{\longrightarrow} v^{2}(e)$. For $k=1, \ldots, \tau-1$ with $M_{0}=0$, we observe

$$
E^{*}\left[\left|\xi_{n, k}^{*}\right|^{2+\delta}\right]=E^{*}\left[\left|\sum_{i=M_{k-1}+1}^{M_{k}} \phi_{i}^{*}(e)\right|^{2+\delta}\right]=E^{*}\left[\left.|| \sum_{i=M_{k-1}+1}^{M_{k}} \frac{1}{\hat{\sigma}} \sqrt{\frac{h}{n}} K_{h, i} \tilde{\sigma}_{g}^{2}\left(\hat{\varepsilon}_{i-1}\right)\left[\hat{\eta}_{i}^{2 *}-\bar{\eta}^{2}\right]\right|^{2+\delta}\right]
$$

which has the same distribution as

$$
\begin{aligned}
& E^{*}\left[\left|\sum_{i=1}^{\min \left\{L_{k}, n\right\}} \frac{1}{\hat{\sigma}} \sqrt{\frac{h}{n}} K_{h, i} \tilde{\sigma}_{g}^{2}\left(\hat{\varepsilon}_{i-1}\right)\left[\hat{\eta}_{i}^{2 *}-\bar{\eta}^{2}\right]\right|^{2+\delta}\right] \\
& =E\left[E^{*}\left(\left|\sum_{i=1}^{\min \left\{L_{k}, n\right\}} \frac{1}{\hat{\sigma}} \sqrt{\frac{h}{n}} K_{h, i} \tilde{\sigma}_{g}^{2}\left(\hat{\varepsilon}_{i-1}\right)\left[\hat{\eta}_{i}^{2 *}-\overline{\eta^{2}}\right]\right|^{2+\delta} \mid L_{k}\right)\right] \\
& =\sum_{\ell=1}^{\infty} \rho(1-\rho)^{\ell-1} E^{*}\left(\left|\sum_{i=1}^{\min \{\ell, n\}} \frac{1}{\hat{\sigma}} \sqrt{\frac{h}{n}} K_{h, i} \tilde{\sigma}_{g}^{2}\left(\hat{\varepsilon}_{i-1}\right)\left[\hat{\eta}_{i}^{2 *}-\overline{\eta^{2}}\right]\right|^{2+\delta}\right) \\
& \leq \frac{1}{\hat{\sigma}^{2+\delta}} \frac{h^{1+\frac{\delta}{2}}}{n^{1+\frac{\delta}{2}}}\left(\frac{1}{h^{2+\delta}} \max _{u \in \mathbb{R}} K^{2+\delta}(u)\right) \sum_{\ell=1}^{\infty} \rho(1-\rho)^{\ell-1} E^{*}\left(\left|\sum_{i=1}^{\min \{\ell, n\}} \tilde{\sigma}_{g}^{2}\left(\hat{\varepsilon}_{i-1}\right)\left[\hat{\eta}_{i}^{2 *}-\bar{\eta}^{2}\right]\right|^{2+\delta}\right) .
\end{aligned}
$$

Thus we consider the order of

$$
\sum_{\ell=1}^{\infty} \rho(1-\rho)^{\ell-1} E^{*}\left(\left|\sum_{i=1}^{\min \{\ell, n\}} \tilde{\sigma}_{g}^{2}\left(\hat{\varepsilon}_{i-1}\right)\left[\hat{\eta}_{i}^{2 *}-\bar{\eta}^{2}\right]\right|^{2+\delta}\right)
$$

which is equal to

$$
\sum_{\ell=1}^{n} \rho(1-\rho)^{\ell-1} E^{*}\left(\left|\sum_{i=1}^{\ell} \tilde{\sigma}_{g}^{2}\left(\hat{\varepsilon}_{i-1}\right)\left[\hat{\eta}_{i}^{2 *}-\bar{\eta}^{2}\right]\right|^{2+\delta}\right)+\sum_{\ell=n+1}^{\infty} \rho(1-\rho)^{\ell-1} E^{*}\left(\left|\sum_{i=1}^{n} \tilde{\sigma}_{g}^{2}\left(\hat{\varepsilon}_{i-1}\right)\left[\hat{\eta}_{i}^{2 *}-\bar{\eta}^{2}\right]\right|^{2+\delta}\right) .
$$

Since $\tilde{\sigma}_{g}^{2}(\cdot)$ is bounded a.s., we have

$$
E^{*}\left(\left|\sum_{i=1}^{\ell} \tilde{\sigma}_{g}^{2}\left(\hat{\varepsilon}_{i-1}\right)\left[\hat{\eta}_{i}^{2 *}-\overline{\eta^{2}}\right]\right|^{2+\delta}\right) \leq M \cdot E^{*}\left(\left|\sum_{i=1}^{\ell}\left(\hat{\eta}_{i}^{2 *}-\overline{\eta^{2}}\right)\right|^{2+\delta}\right)=O_{p}\left(\ell^{1+\frac{\delta}{2}}\right)
$$

for some positive $M$, where the last equality for $O_{p}$-term is a well-known fact in references on the stationary bootstrap like Politis and Romano (1994) or Hwang and Shin (2012). Thus the first term in (A.4) becomes $O_{p}\left(\sum_{\ell=1}^{n} \rho(1-\rho)^{\ell-1} \ell^{1+\delta / 2}\right)$ and the second term in (A.4) becomes $O_{p}\left(\sum_{\ell=n+1}^{\infty} \rho(1-\right.$ $\left.\rho)^{\ell-1} n^{1+\delta / 2}\right)$, which is less than $O_{p}\left(\sum_{\ell=n+1}^{\infty} \rho(1-\rho)^{\ell-1} \ell^{1+\delta / 2}\right)$. Thus, (A.4) is of order $O_{p}\left(1 / \rho^{1+\delta / 2}\right)$ using the identity $\sum_{\ell=1}^{\infty}(1-\rho)^{\ell-1} \ell^{a}=O\left(1 / \rho^{a+1}\right)$ for $a \geq 1$. 
Hence $E^{*}\left[\left|\xi_{n, k}^{*}\right|^{2+\delta}\right]=O_{p}\left(1 /(n \rho h)^{1+\delta / 2}\right)$ and thus the left term in (A.3) is of order $O_{p}\left(\tau /(n \rho h)^{1+\delta / 2}\right)$. Since $\tau=n \rho+O_{p}(\sqrt{n \rho})$ by Politis and Romano (1994), the left term in (A.3) is of order $O_{p}\left(1 /\left((n \rho h)^{\delta / 2}\right.\right.$ $h)$ ), which tends to zero for choosing $\rho$ such that $n \rho h^{1+2 / \delta} \rightarrow \infty$. Therefore, the convergence in probability in (A.3) holds and the desired asymptotic normality of $\sum_{i=1}^{n} \phi_{i}^{*}(e)$ is established.

\section{References}

Bossaerts, P., Hafner, C. and Hardle, W. (1996). A new method for volatility estimation with applications in foreign exchange rate series. In G. Bol, G. Nakhaeizadeh, and K. H. Vollmer (Eds.), Finanzmarktanalyse und -prognose mit innovativen quantitativen Verfahren (71-83), PhysicaVerlag, Heidelberg.

Franke, J., Kreiss, J.-P. and Mammen, E. (2002). Bootstrap of kernel smoothing in nonlinear time series, Bernoulli, 8, 1-37.

Franke, J., Neumann, M. H. and Stockis, J. P. (2004). Bootstrapping nonparametric estimators of the volatility function, Journal of Econometrics, 118, 189-218.

Goncalves, S. and Kilian, L. (2007). Asymptotic and bootstrap inference for AR( $\infty)$ processes with conditional heteroscedasticity, Econometric Reviews, 26, 609-641.

Hafner, C. (1998). Nonlinear Time Series Analysis with Applications to Foreign Exchange Rate Volatility, Physica Verlag, Heidelberg.

Hardle, W. and Tsybakov, A. (1997). Local polynomial estimation of the volatility function in nonparametric autoregression, Journal of Econometrics, 81, 223-242.

Hwang, E. and Shin, D. W. (2011). Stationary bootstrapping for non-parametric estimator of nonlinear autoregressive model, Journal of Time Series Analysis, 32, 292-303.

Hwang, E. and Shin, D. W. (2012). Stationary bootstrap for kernel density estimators under $\psi$-weak dependence, Computational Statistics and Data Analysis, 56, 1581-1593.

Hwang, E. and Shin, D. W. (2013a). Stationary bootstrapping realized volatility under market microstructure noise, Electronic Journal of Statistics, 7, 2032-2053.

Hwang, E. and Shin, D. W. (2013b). Stationary bootstrapping realized volatility, Statistics \& Probability Letters, 83, 2045-2051.

Kreiss, J.-P., Neumann, M. H. and Yao, Q. (2008). Bootstrap tests for simple structures in nonparametric time series regression, Statistics and Its Interface, 1, 367-380.

Kreiss, J.-P. and Paparoditis, E. (2011). Bootstrap methods for dependent data: A review, Journal of the Korean Statistical Society, 40, 357-378.

Paparoditis, E. and Politis, D. N. (2005). Bootstrapping unit root tests for autoregressive time series, Journal of the American Statistical Association, 100, 545-553.

Parker, C., Paparoditis, E. and Politis, D. N. (2006). Unit root testing via the stationary bootstrap, Journal of Econometrics, 133, 601-638.

Politis, D. N. and Romano, J. P. (1994). The stationary bootstrap, Journal of the American Statistical Association, 89, 1303-1313.

Robinson, P. M. (1983). Nonparametric estimation for time series models, Journal of Time Series Analysis, 4, 185-208.

Shimizu, K. (2013). The bootstrap does not always work for heteroscedastic models, Statistics \& Risk Modeling, 30, 189-204.

Shimizu, K. (2014). ). Bootstrapping the nonparametric ARCH regression model, Statistics \& Probability Letters, 87, 61-69. 
Shin, D. W. and Hwang, E. (2013). Stationary bootstrapping for cointegrating regressions, Statistics \& Probability Letters, 83, 474-480.

Swensen, A. R. (2003). Bootstrapping unit root tests for integrated processes, Journal of Time Series Analysis, 24, 99-126.

Received May 19, 2015; Revised July 29, 2015; Accepted July 29, 2015 
